\title{
Parietal Plasmacytoma: An Ominous Sign of Disease Recurrence
}

\author{
Narjust Duma MD ${ }^{1, *}$, Dhruvesh Patel ${ }^{2}$, Thayer Nasereddin ${ }^{2}$ \\ ${ }^{1}$ Rutgers-New Jersey Medical School, Newark, New Jersey, 07103, United States \\ ${ }^{3}$ Saint George's University, True blue, 38901, Grenada \\ *Corresponding author: narjustperezmd@gmail.com
}

Received April 29, 2015; Revised May 09, 2015; Accepted May 15, 2015

\begin{abstract}
Plasmacytomas located in the central nervous system are rareentities; with no pathognomonic clinical or radiologic features histology is required for a definite diagnosis. We present a case of a dural plasmacytoma as the manifestation of disease recurrence in a patient with systemic multiple myeloma. 51 year old male with history of IgG kappa myeloma, who underwent complete remission after an autologous bone marrow transplant presented to the clinic 10 months post-remission complaining of a "head mass". On physical examination, he was found to have a large soft mass in the parietal area. On MRI the lesion was described as an extra-axial mass, $5.5 \mathrm{~cm} \mathrm{x5cm}$ diameter, with diffuse enhancement of the dura. Biopsy of the mass revealed anextramedullary plasmacytoma. At that time, his $\mathrm{M}$ spike and immunoglobulins levels were unremarkable. Several months after the presentation of the parietal mass, the patient developed recurrence of disease. He underwent a second autologous bone marrow transplant that markedly improved the size of the plasmacytoma. Patient achieved a complete remission after the second transplant. However, 6 months later the dural plasmacytoma increased in size and once again and his myeloma markers remained within normal levels. Unfortunately, recurrence of the multiple myeloma occurred several months later and despite several cycles of chemotherapy, the patient continued to have progression of disease. He then decided for a more conservative treatment and was sent to home hospice. Central nervous system plasmacytomas can present as solitary lesions or as part of systemic multiple myeloma, these tumors tend to have a direct relationship with the activity of the disease and can present as an early sign of recurrence despite normal multiple myeloma markers on peripheral blood.
\end{abstract}

Keywords: dural plasmacytoma, multiple myeloma, solitary plasmacytoma, multiple myeloma recurrence

Cite This Article: Narjust Duma MD, Dhruvesh Patel, and Thayer Nasereddin, "Parietal Plasmacytoma: An Ominous Sign of Disease Recurrence.” American Journal of Medical Case Reports, vol. 3, no. 6 (2015): 163-166. doi: 10.12691/ajmcr-3-6-4.

\section{Background}

Plasma cell neoplasms can be broadly categorized into multiple myeloma and plasmacytomas. Multiple myeloma, the more common form of plasma cell neoplasms, is composed of abnormal plasma cells that proliferate in the bone marrow. These abnormal cells disrupt normal hematopoiesis, invade adjacent bone and produce paraproteins. Anemia, hypercalcemia, and renal insufficiency are the consequent symptoms of this systemic disease. In contrast to multiple myeloma, plasmacytomas are composed of a mass of neoplastic plasma cells. These masses can be isolated neoplasms with or without the systemic symptoms of multiple myeloma.

Plasmacytomas are further subdivided inthree distinct types of plasmacytomas-solitary plasmacytoma of the bone, extramedullary plasmacytoma and multiple plasmacytomas that are either primary or recurrent [1]. Extramedullary plasmacytomas account for 3\% of plasma cell neoplasms. These arise outside the bone marrow and can be present before the diagnosis of multiple myeloma or at any stage of the disease. These extramedullary plasma cell neoplasms are most commonly seen in the respiratory tract followed by gastrointestinal tract, central nervous system, bladder, breast, testis and lymph nodes $[2,3,4]$. Central nervous system plasmacytomas are very rare; as per our literature review less than 20 cases have been reported in the English literature [5-11]. Here we present our experience with a dural plasmacytoma as the initial sign of disease recurrence in a patient with systemic multiple myeloma.

\section{Case Presentation}

51 year old Haitian male with past medical history of IgG kappa, Durie-Salmon stage III and International Staging System stage IImultiple myeloma that initially presented with compression fracture of L1 and lytic lesions in the central portion of L5. At time of diagnosis the patient had pancytopenia with white blood cell count of $3.2 \times 10^{3} / \mu \mathrm{L}$, hemoglobin $5.7 \mathrm{~g} / \mathrm{dL}$, platelets of $122.000 / \mu \mathrm{L}$, blood urea nitrogen $12 \mathrm{mg} / \mathrm{dL}$,creatinine of $1.4 \mathrm{mg} / \mathrm{dL}$ and calcium of $8.7 \mathrm{mg} / \mathrm{dL}$. Further testing 
revealed IgG 10,325 mg/dL, IgA 35 mg/dL, IgM 3 mg/dL, and serum M component of $10.11 \mathrm{~g} / \mathrm{L}$, with an IgG kappa monoclonal protein. Imaging studies showed unremarkable cervical and thoracic spine and normal brain magnetic resonance imaging (MRI). Bone marrow biopsy at that time revealed sheets of plasma cell with $90 \%$ of the marrow space involved. Flow cytometry showed 55\% plasma cells with cytogenetics notable for positive translocation $\mathrm{t}(4: 14)$ in $46.5 \%$ of the cells, which helped classify the neoplasm as an intermediate risk myeloma.

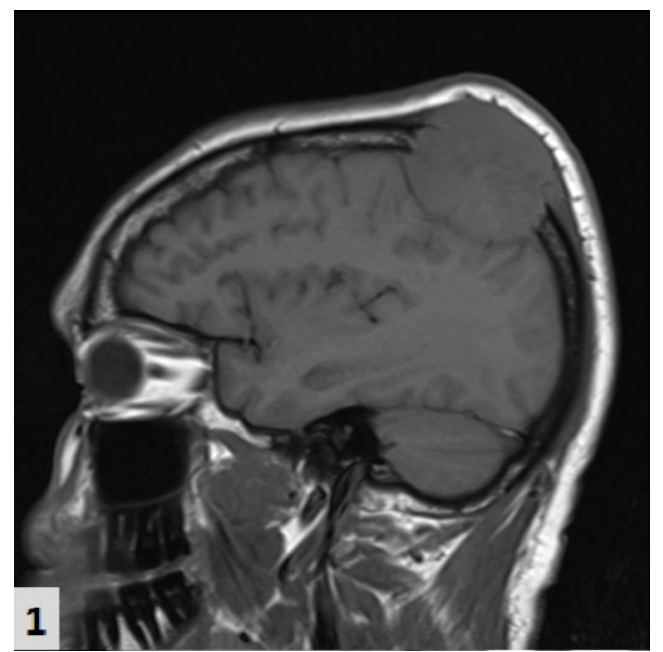

Figure 1. Brain MRIsagittal view, revealed an extra-axial mass located in the parietal calvarium measuring $5.5 \times 5 \mathrm{~cm}$



Figure 2. Brain MRI showed a parietal mass with enhancement of the adjacent dura and displacement of the right parietal lobe.

After the initial diagnosis the patient completed several cycles of chemotherapy including bortezomib and lenalidomide until he achieved partial response; seven months later he underwent anautologous bone marrow transplant receiving a total of 4.92 million CD34-positive cells $/ \mathrm{kg}$. His transplant had no complications and the patient achieved a very good partial response (VGPR) (serum and urine M-protein were detectable by immunofixation but not on electrophoresis) for 10 months. He then presented to the oncology clinic complaining of a "head mass". During physical examination, a right parietal soft, painless mass measuring approximately $6 \mathrm{~cm}$ was observed; the lesion was attached to the skull and covered with intact skin. His multiple myeloma markers were unremarkable. Brain MRI revealed a $5.5 \mathrm{~cm}$ extra-axial mass along the right parietal calvarium with diffuse enhancement of the dura (Figure 1-Figure 2). Patient underwent a computed tomography (CT)-guided biopsy of the mass as well as whole body positron emission tomography (PET)-scan, the latter revealed mild hypermetabolism in multiple bone lesions, including a right fronto-parietal lytic lesion with a diameter of $5.5 \times 4.7 \mathrm{~cm}$, lesions were also observed in the $\mathrm{C} 2$ vertebral body, L1 to L5, pelvis and left proximal femur.

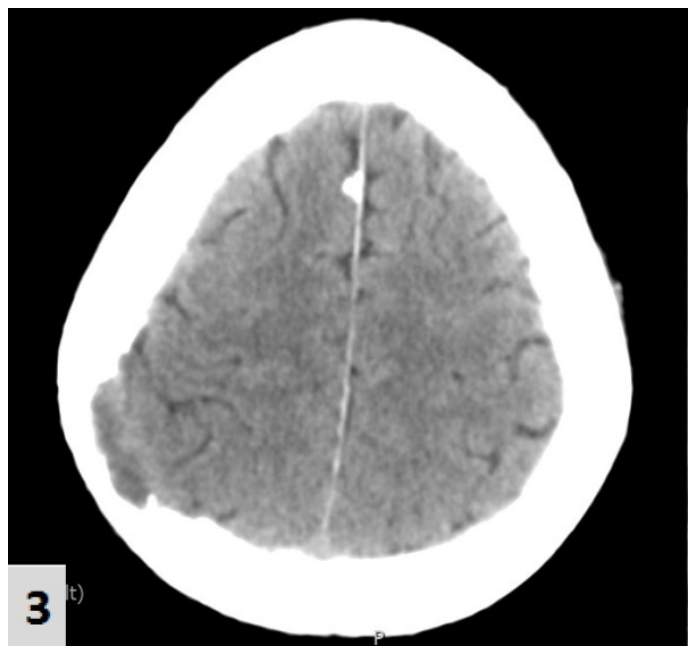

Figure 3. Post-bone marrow transplant head CT revealed asignificant decrease in size of themass in the right parietal bone.

Histology from the parietal mass revealed a malignant plasma cell neoplasm; tumor cells were CD138 positive with kappa light chain restriction. At that time patient was offered radiation therapy for the treatment of the parietal plasmacytoma, he refused radiation due to an upcoming religious trip and plans to pursue of alternative medicine in his native country. Two months after the initial presentation of the parietal lesion, the patient came back to our clinic and this time, peripheral blood showed recurrence of the disease with quantitative immunoglobulins revealing an IgG of $1977 \mathrm{mg} / \mathrm{dL}$, IgA of $135 \mathrm{mg} / \mathrm{dL}$, IgM of $17 \mathrm{mg} / \mathrm{dL}$ and a M spike of $1.28 \mathrm{~g} / \mathrm{dL}$. Serum free kappa light chain measured $116.25 \mathrm{mg} / \mathrm{L}$ with a serum free lambda light chain of $7.53 \mathrm{mg} / \mathrm{L}$. The patient was then started on a combination of carfilzomib, cyclophosphamide and dexamethasone. After 3 cycles of chemotherapy, heachieved partial response with improvement of his $\mathrm{M}$ spike(0.17). He underwent a second autologous bone marrow transplant, again receiving 4.9 million CD34-positive cells. One week after transplantation the parietal mass markedly decreased in size with head CTreporting significant decrease in conspicuity of the soft tissue mass in the right parietal bone, now measuring 3.8 × $2.4 \mathrm{~cm}$ (Figure 3). Patient' smyeloma markers remained low. Five months after the second autologous bone marrow transplant, thepatient started experiencing increasing size of the parietal mass. Follow up brain MRI revealed recurrent parietal plasmacytoma this time measuring $5.9 \mathrm{~cm}$ anterior posterior x $6.7 \mathrm{~cm}$ transverse $\mathrm{x} 3.4 \mathrm{~cm}$ craniocaudal (Figure 4). Due to several recurrences with previous regimes, the treatment team discussed with the patient the possibility of enrolling in a clinical trial. During this period of discussion, the patient received treatment with pomalidomide. In the 2 months that followed, the patient 
continued to have progression of disease with increasing size of the parietal plasmacytoma. After careful consideration the patient and family decided for a more conservative management and the patient was sent to home hospice.

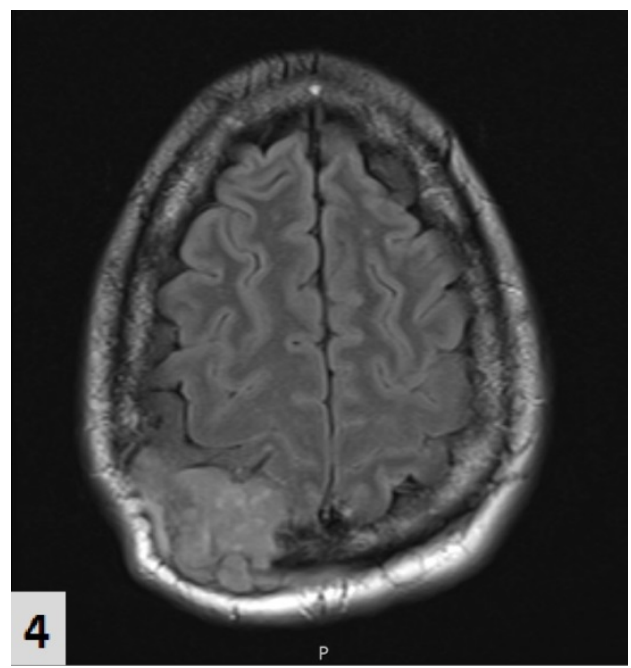

Figure 4. Follow up brain MRI showed a recurrent plasmacytoma that developed in previously treated parietal region. Enlarged compared to initial MRI, now measuring $5.9 \mathrm{~cm}$ x $6.7 \mathrm{~cm}$.

\section{Discussion}

Central nervous system (CNS) plasmacytomas are extremely rare, even in the presence of multiple myeloma. In our case, the plasmacytoma presented after the first autologous bone marrow transplant without initial elevation of immunoglobulins. Its behavior, specially the progressive increase in size over weeks and months, can be described as the preceding signs of disease recurrence.

There are three varieties of plasma cell tumors, including multiple myeloma, solitary plasmacytoma of the bone, and extramedullary plasmacytomas. Multiple myeloma represents systemic disease while the other two represent local forms of plasma cell neoplasms. The interrelationship of these tumors remains unclear. Some authors have suggested that they are variants of the same disease [12]. CNS plasmacytomas are very unique entities and can present as solitary tumors or in conjunction with systemic multiple myeloma. The true nature of extramedullary plasmacytoma is still controversial. Some authors believeextramedullary plasmacytomas arise from B-type lymphocytes that originate in the bone marrow, migrate to the peripheral lymphoid tissues, and then undergo plasmacytoid differentiation [13]. They further postulated that the antigenic stimulation of these tissues by chronic infection may influence these tissues to be predisposed to develop such tumors [13].

Extramedullary plasmacytomas can be considered true solitary plasmacytoma when there are not clonal plasma cells in the bone marrow. The international myeloma working group (IMWG) recommends the term "solitary plasmacytoma with minimal marrow involvement" if bone marrow clonal cells are present but less than 10 percent [14]. Patients with a single extramedullary plasmacytomas who have 10 percent or more clonal bone marrow plasma cells, on the other hand, will be considered as having multiple myeloma.
The international myeloma working group has also established a list of diagnostic criteria for extramedullary plasmacytomas, including: tissue biopsy of the lesion showing monoclonal plasma cell histology, no M-protein in serum and/or urine, bone marrow plasma cell infiltration not exceeding 5 percent or all nucleated cells, and absence of osteolytic bone lesions, hypercalcemia, renal failure or other tissue involvement $[14,15,16]$. Extramedullary plasmacytomas incidence in males is three times higher than in females, with an average age of 57 years (range: 50-70 years) at the time of diagnosis [17]. In $80 \%$ of the cases, extramedullary plasmacytomas are located in the upper respiratory tract; more rarely they affect the gastrointestinal tract, lymph nodes, bladder, and CNS [18].

CNS plasmacytomas have no pathognomonic clinical or radiologic features, so definite diagnosis requires histopathological examination of the tumor. These are dense, homogenous tumors with infiltrates of plasma cells. Amyloid deposition may be present in 15 to $38 \%$ of the cases. The most common immunoglobulin expressed by these tumors is IgG with kappa chain restriction. Plasmacytomas can be graded low (grade 1), intermediate (grade 2) and high grades (grade 3) based on the cellular atypia [17]. The differential diagnosis in these patients should include plasma cell granulomas; these can be distinguished from plasmacytomas microscopically. Histologically, plasma cell granulomas are characterized by an admixture of plasma cells, lymphocytes, or macrophages on a background of partly necrotic tissue, including some areas of fibrosis and other areas of granulation tissue [19]. Other differential diagnosis to take into account include: meningioma, metastasis, lymphoma, dural sarcoma and leptomeningeal carcinomatosis [20].

Some typical findings of dural plasmacytomas include isodensity or hyperdensity on CT scan as well as T1 equal to high signal intensity and T2 markedly hypointense signal on MRI. In our case the mass was described as large robustly enhancing extra-axial mass centered along the right parietal calvarium, measuring $5.5 \times 5$ centimeters, with slightly restricted diffusion and mild enhancement of the adjacent dura. In previous studies, plasmacytomas of the skull have been described as slightly inhomogeneous, expansible masses eroding the bone that is isointense to the brain on non-contrast brain CT scans [21,22].

The treatment of CNS plasmacytomas changes according to the extent of the disease, in solitary tumors, local surgical debulking followed by radiotherapy have shown good outcomes but once the disease becomes systemic, chemotherapy is the main treatment [23,24,25]. It has been reported that extramedullary localizations of myeloma originating from the cranial bone usually respond well to new drugs (e.g., thalidomide, bortezomid and lenalidome) compared tointraparenchyma plasmacytomas [26].

In conclusion, we present a case of a central nervous plasmacytoma in a patient with multiple myeloma, the unique characteristics of this case include: the central nervous system location of the plasmacytoma and the tumor's behavior. Tumor growth was directly associated with recurrence of disease before the increase of immunoglobulins levels could be detected in peripheral blood. Central nervous plasmacytomas are very rare entities, these can present as solitary tumors or as one of 
the manifestations of multiple myeloma. These tumors do not have any specific clinical or radiologic features and histology is required for a definite diagnosis. Surgery and radiation are the preferred treatment in the case of solitary tumors and when the lesions are accompanied of systemic multiple myeloma the cornerstone of treatment is cytotoxic therapy.

\section{Acknowledgement}

We would like to acknowledge our mentors for their collaboration during the preparation of this manuscript.

\section{References}

[1] "Criteria for the Classification of Monoclonal Gammopathies, Multiple Myeloma and Related Disorders: A Report of the International Myeloma Working Group." British Journal of Haematology 121.5, 2003: 749-57.

[2] Ferrari, S., C. Tecchio, G. Turri, S. Richelli, C. Ghimenton, S Monaco, and G. Todeschini. "Unusual Case of Solitary Intraparenchymal Brain Plasmacytoma." Journal of Clinical Oncology 2012; 30.33: E350-352.

[3] Senapati SB, Mishra SS, Dhir MK, Das S, Tripathy K. A case of multiple myeloma presenting as scalp swelling with intracranial extension. Journal of Neurosciences in Rural Practice. 2013;4(4):445-448.

[4] Daghighi MH, Poureisa M, Shimia M, Mazaheri-Khamene R, Daghighi S. Extramedullary Plasmacytoma Presenting as a Solitary Mass in the Intracranial Posterior Fossa. Iranian Journal of Radiology. 2012; 9(4):223-226.

[5] Eum JH, Jeibmann A, Wiesmann W, Paulus W, Ebel H. Multiple myeloma manifesting as an intraventricular brain tumor. J Neurosurg. 2009; 110:737-739.

[6] Cerase A, Tarantino A, Gozzetti A, Muccio CF, Gennari P, Monti L, Di Blasi A, Venturi C. Intracranial involvement in plasmacytomas and multiple myeloma: a pictorial essay. Neuroradiology. 2008; 50:665-674.

[7] Wavre A, Baur AS, Betz M, Muhlematter D, Jotterand M, Zaman $\mathrm{K}$, Ketterer N. Case study of intracerebral plasmacytoma as an intial presentation of multiple myeloma. Neuro Oncol.2007; 9: 370-372.

[8] Higurashi M, Yagishita S, Fujitsu K, Kitsuta Y, Takemoto Y, Osano S. Plasma cell myeloma of the skull base: report of two cases. Brain Tumor Pathol. 2004; 21:135-141.
[9] Mantyla R, Kinnunen J, Bohling T. Intracranial plasmacytoma: a case report. Neuroradiology 1996; 38:646-649.

[10] Coppeto JR, Monteiro ML, Collias J, Uphoff D, Bear L. FosterKennedy syndrome caused by solitary intracranial plasmacytoma. Surg Neurol. 1983; 19:267-272.

[11] Trimble GX. Plasmacytoma primary in the brain. New Eng J Med. 1973; $15: 582$

[12] Webb HE, Harrison EG, Masson JK, ReMineWH: Solitary extramedullary myeloma (plasmacytoma) of the upper part of the respiratory tract and oropharynx. Cancer 1962 15:1142-1155.

[13] Wisniewski T, Sisti M, Inhirami G, Knowles DM, Powers JM: Intracerebral solitary plasmacytoma. Neurosurgery 1990 27: 826829.

[14] Kyle, R. A., and S. V. Rajkumar. "Criteria for diagnosis, staging, risk stratification and response assessment of multiple myeloma." Leukemia 23.1 (2009): 3-9.

[15] Durie, B. G. M., et al. "International uniform response criteria for multiple myeloma." Leukemia 20.9 (2006): 1467-1473.

[16] Greipp, Philip R., et al. "International staging system for multiple myeloma." Journal of Clinical Oncology 23.15 (2005): 3412-3420.

[17] Attanasio G, Viccaro M, Barbaro M, De Seta E, Filipo R. Extramedullary plasmacytoma of paranasal sinuses. A combined therapeutic strategy. ActaOtorhinolaryngologicaItalica. 2006; 26(2):118-120.

[18] Galieni P, Cavo M, Pulsoni A, et al: Clinical outcome of extramedullary plasmacytoma. Haematologica 2000, 85:47-51.

[19] Bahadori M, Liebow AA: Plasma cell granulomas of the lung. Cancer, 1973, 31:191-208.

[20] Cerase A, Tarantino A, Gozzetti A, Muccio CF, Gennari P, Mont $\mathrm{L}$, et al. Intracranial involvement in plasmacytomas and multiple myeloma: a pictorial essay. Neuroradiology. 2008; 50(8):665-74.

[21] Rahmah N, Brotoarianto H, Andor E, Kusnarto G, Muttaqin Z, Hongo K. Dural plasmacytoma mimicking meningioma in a young patient with multiple myeloma. Biomed Imaging Interv J. 2009; 5(2).

[22] Provenzale JM, Schaefer P, Traweek ST, Ferry J, Moore JO, Friedman AH, et al. Craniocerebral plasmacytoma: MR features. AJNR Am J Neuroradiol. 1997; 18(2):389-92.

[23] Engelsma RJ, De Bree R, Janssen JJ, Scheeren RA. Plasmacytoma of the mastoid bone: Solitary and systemic. J Laryngol Otol. 2000 114:378-80.

[24] LorsbachRB, Hsi ED, Dogan A, Fend F. Plasma cell myeloma and related neoplasms. Am J ClinPathol. 2011; 136:168-82.

[25] Kyle RA, Therneau TM, RajkumarSV, Larson DR, Plevak MF, Melton LJ., 3 rd Incidence of multiple myeloma in Olmsted County, Minnesota: Trend over 6 decades. Cancer. 2004; 101:2667-74.

[26] Gozzetti A, Cerase A, Lotti F, Rossi D, Palumbo A, Petrucci MT, et al. Extramedullary intracranial localization of multiple myeloma and treatment with novel agents: A retrospective survey of 50 patients. Cancer. 2012; 118:1574-84. 